Methods We performed a randomised, cross-over trial in 21 mildto-moderate persistent asthmatics receiving ICS with elevated FeNO $(>30 \mathrm{ppb})$ that increased further $(>10 \mathrm{ppb})$ after ICS washout. Patients were randomised to 2 weeks of either fluticasone propionate $50 \mu \mathrm{g}$ twice-daily (FP100) or $250 \mu \mathrm{g}$ twice-daily (FP500). The primary outcome was response in diurnal domiciliary FeNO levels. Secondary outcomes included: mannitol challenge; serum eosinophilic cationic protein (ECP); blood eosinophil count; and asthma control questionnaire (ACQ).

Results We found significant dose-related reductions of diurnal FeNO compared to baseline - morning FeNO: baseline $=71 \mathrm{ppb}$ (95\%CI:61-83ppb); FP100=34ppb (95\%CI:29-40ppb), p <0.001; FP500 $=27 \mathrm{ppb}$ (95\%CI:22-33ppb), p<0.001; and significant dose separation for morning, $p<0.05$, and evening, $p<0.001$. Time series FeNO displayed exponential decay (Figure 1): FP100 R² $=0.913$, halflife $=69 \mathrm{hrs}$ (95\%CI:50-114hrs); FP500 R²=0.966, half-life $=55 \mathrm{hrs}$ (95\%CI:45-69hrs); as well as diurnal variation. ACQ showed significant improvements exceeding the minimal important difference $(>0.5)$ with values in keeping with controlled asthma $(<0.75)$ after each dose: FP100 $=0.48$ (95\%CI:0.24-0.71), p $=0.004 ;$ FP500 $=0.37$ (95\%CI:0.18-0.57), $p=0.001$. All other secondary inflammatory related outcomes (mannitol, ECP and eosinophils) showed significant improvements from baseline but no dose separation.

Conclusions There is a significant dose-response of diurnal FeNO to ICS in asthmatics with an elevated FeNO phenotype, which translates into well-controlled asthma. Further interventional studies are warranted using domiciliary $\mathrm{FeNO}$ in this specific phenotype.

\section{S7 INFLUENCE OF BETA-2 ADRENOCEPTOR GENOTYPE ON RESPONSE TO REGULAR RACEMIC OR LEVOSALBUTAMOL IN STEROID TREATED ASTHMATICS}

doi:10.1136/thoraxjnl-2012-202678.013

WJ Anderson, PM Short, PA Williamson, AE Morrison, CNA Palmer, R Tavendale, BJ Lipworth. University of Dundee, Dundee, United Kingdom

Background Asthmatic patients receiving inhaled corticosteroids often take frequent add-on therapy with albuterol despite ondemand prescription. We wished to evaluate trough methacholine airway hyper-responsiveness (the primary outcome) following regular treatment with racemic salbutamol and levosalbutamol compared to placebo, in steroid treated asthmatics stratified according to beta-2 adrenoceptor 16 genotype.

Methods We performed a randomised, double-blind, placebocontrolled, triple crossover trial comparing 2 weeks of regular ther-

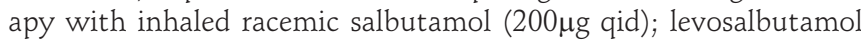
$\left(100 \mu \mathrm{g}\right.$ qid); or placebo on methacholine $\mathrm{PC}_{20} 6$ hours post dose in 30 persistent asthmatics (15 homozygous Arg16 and Gly16) receiving inhaled corticosteroids.

Results There was no rebound worsening of trough airway hyperresponsiveness to methacholine after chronic exposure to either racemic $(p=0.53)$ or levosalbutamol $(p=0.84)$ compared to placebo; nor between genotypes - as doubling dilution (dd) difference in methacholine $\mathrm{PC}_{2} 0$ from placebo (Figure 1): salbutamol/Arg16= $0.36 \mathrm{dd}$ (95\% CI: -0.43 , 1.15); salbutamol/Gly16=0.01dd (95\% CI: -0.47, 0.49); levosalbutamol/Arg16=-0.01dd (95\% CI: $-0.89,0.87$ ); levosalbutamol/Gly16 $=0.28 \mathrm{dd}(95 \% \mathrm{CI}:-0.22,0.77)$. Both active treatments improved morning PEF in Gly16 $(p=0.04)$ but not Arg16 patients $(p=0.50)$; while evening PEF improved in both Gly16 $(p<0.001)$ and Arg16 patients $(p=0.006)$.

Conclusions Chronic exposure to either racemic or levosalbutamol added to inhaled corticosteroids did not cause rebound worsening of airway hyper-responsiveness at trough compared to placebo; with no difference seen between beta-2 adrenoceptor 16 genotypes.

\section{S8 CAN EOSINOPHIL AND NEUTROPHIL MIGRATION BE THE KEY TO PHENOTYPING ASTHMA?}

doi:10.1136/thoraxjn-2012-202678.014

'JJ Lukawska, 'L Livieratos, 'B Sawyer, 'T Lee, 'M O'Doherty, 'M Kofi, 'J Ballinger, ${ }^{2} \mathrm{G}$ Gnanasegeran, ${ }^{2} \mathrm{E}$ O'Young, ' $\mathrm{C}$ Corrigan, 'G Mullen; ${ }^{1} \mathrm{King}$ 's College London, London, UK; 'uy's and St Thomas' Hospital NHS Foundation Trust, London, UK

Introduction To date, our knowledge of in vivo migration of neutrophils and eosinophils in homeostasis and disease states is based on granulocytes. Here we present a pilot study using purified human eosinophils or neutrophils and demonstrate their differential in vivo kinetics in asthmatic and healthy volunteers. Methods: On two separate occasions $100 \mathrm{ml}$ of blood was obtained from eight human

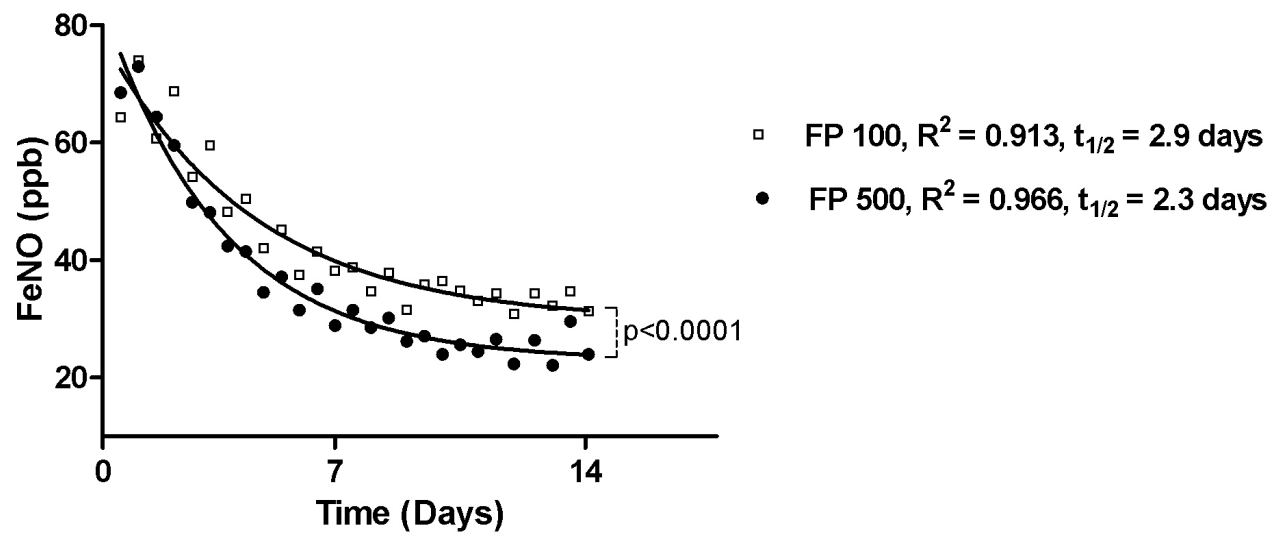

Time series morning and evening exhaled tidal nitric oxide (FeNO) values and one-phase exponential decay curves. FeNO values displayed as geometric means at each sequential time point for each group. $R^{2}=$ coefficient of determination (goodness of fit) of exponential decay curves to each data set. $t_{1 / 2}=$ half-life of exponential decay. $p p b=$ parts per billion .

Abstract S6 Figure 1 